\title{
Zelluläre und Antikörper-basierte Immuntherapien der Leukämie
}

Die akute Leukämie ist die häufigste Krebserkrankung im Kindes- und Jugendalter. In den vergangenen Jahrzehnten konnten die Gesamtüberlebensraten von Patienten mit akuter lymphoblastischer Leukämie (ALL) auf über $80 \%$ erhöht werden. Die Prognose von Patienten mit akuter myeloischer Leukämie (AML) ist schlechter. Primär refraktäre oder rezidivierende ALL bleibt mit 40-60\% Überlebensrate jedoch prognostisch unbefriedigend [1]. Risikofaktoren der ALL sind in den verschiedenen Protokollen klinische und genetische Marker sowie Therapieansprechen (Prednisonresponse, MRD [minimal residual disease]). Marker der Immuninteraktion sind für die Prognoseeinschätzung der pädiatrischen ALL bislang nicht bekannt. Akute Leukämien gelten als niedrig immunogene Erkrankungen. Sie sind als Bestandteil des Immunsystems häufig selbst nicht sichtbar für das Immunsystem. Durch Antikörper oder Zellinfusionen kann die Hürde jedoch überwunden werden. Der bispezifische T-Zell-Engager Blinatumomab (Anti-CD3/-CD19) ist ein synthetisches Molekül aus variablen Antikörperfragmenten, welches jede T-Zelle des Körpers zu einer Reaktion gegen Leukämiezellen zwingt (sofern diese CD19 auf der Oberfläche tragen). Mit dem Start des IntReALL-Protokolls kommt seit 2015 bei Patienten mit rezidivierter ALL vor allogener Stammzelltransplantation Blinatumomab zum Einsatz. CAR-T-Zellen sind genetisch modifizierte T-Zellen, welche einen chimären Antigenrezeptor (CAR) auf der Oberfläche tragen. Dieser besteht aus einer extrazellulären Domäne eines Antikörperbindungsfragmentes und einer intrazellulären CD3zeta-Kette mit Co-Stimulation. Seit 2017 sind in den USA CAR-T-Zellen gegen die ALL als Therapie zugelassen. Seit Sommer 2018 ist diese Therapie auch in Deutschland zugelassen. Die pädiatrische AML ist bislang unzureichend untersucht auf die Möglichkeiten der Immuntherapie.

\section{Leukämie und Immunsystem}

Maligne Zellen sind in der Lage, das Immunsystem aktiv über die Expression co- stimulatorischer oder co-inhibitorischer Checkpointmoleküle zu modifizieren [2, 3]. Bei anderen Krebserkrankungen wie dem malignen Melanom konnte bereits gezeigt werden, dass eine Überexpression inhibitorischer Checkpointmoleküle auf der Oberfläche der Tumor- und der korrespondierenden Immunzellen mit einer schlechteren Prognose assoziiert ist und eine Therapie mit den entsprechenden Checkpointinhibitoren eine vielversprechende Alternative darstellt $[4,5]$. Für die pädiatrische akute lymphatische Leukämie wurden bislang keine systematischen Studien durchgeführt, um die Relevanz immunmodulatorischer Checkpointmoleküle für Prognose und Therapie der Erkrankung zu beleuchten.

\section{Immuntherapie der ALL}

Neben der allogenen Stammzelltransplantation zeigen neue immuntherapeutische Ansätze für die Behandlung der akuten lymphatischen Leukämie wegweisende Erfolge. Allerdings bleibt bislang ungeklärt, warum manche ALL-Patienten auf die TZelltherapien ansprechen und wiederum andere resistent sind.

Der CD3-CD19-bispezifische T-Zell-Engager (BiTE) Blinatumomab erhielt im Dezember 2014 die FDA-Zulassung, nachdem er große Erfolge in Phase I/II-Studien bei der Behandlung von rezidivierenden oder refraktären Non-Hodgkin-Lymphomen (NHL) und akuten lymphatischen Leukämien aufweisen konnte [6].

Im Feld des adoptiven Zelltransfers zeigen gentechnisch veränderte T-Zellen mit chimärem Antigenrezeptor (CAR-T-Zellen) eine signifikante Steigerung des Eventfree-Survivals bei Patienten nach Zweitoder Drittlinientherapie. Gegen CD19 gerichtete Zweitgenerationen-CAR-T-Zellen, die neben der antigenbindenden Domäne co-stimulatorische Abschnitte wie CD3zeta und CD28 oder 4-1BB beinhalten, werden aktuell in über 15 US-amerikanischen Phase-I/II-Studien evaluiert und zeigen bei primär refraktärer oder mehrfach $(\geq 2)$ rezidivierter pädiatrischer ALL ein Therapie- ansprechen von 70 bis $93 \%$ [7,8]. Kürzlich erfolgte die Zulassung von zwei gegen CD19 gerichteten CAR-T-Zell-Präparaten durch die FDA und die EMA.

Antikörper, welche inhibitorische Signale der Tumorzellen blockieren und damit eine T-Zellantwort gegen Tumorzellen verstärken, nennt man Checkpointinhibitoren. Diese wurden bislang im Wesentlichen bei soliden Tumoren eingesetzt. Eine Überexpression der co-inhibitorischen Moleküle CTLA-4 und PD-1/-L1 im Tumor ist mit einer schlechten Prognose assoziiert. CTLA-4 ist auf T-Zellen exprimiert und konkurriert mit CD28 um die Bindung von CD80 und CD86. Bindet CTLA-4 an CD80/ 86 , wird nicht ein stimulierendes, sondern ein inhibierendes Signal an die T-Zelle vermittelt. PD-1 ist ebenfalls auf T-Zellen exprimiert und löst bei Bindung an seine Liganden PD-L1 oder PD-L2 eine Inhibierung der T-Zelle aus. Antikörper gegen PD-1 (Pembrolizumab, Nivolumab) und CTLA-4 (Ipilimumab) zeigten in Phase I-/II-Studien vielversprechende Ansprechraten bei Patienten mit malignem Melanom, Bronchialkarzinom u.a. [4, 5]. In der Zwischenzeit wurde eine Vielzahl weiterer Immuncheckpointmoleküle identifiziert (unter ihnen TIM-3, Lag-3 und BTLA). Für einige von ihnen werden bereits Antikörper für die klinische Anwendung entwickelt. In der Behandlung der akuten lymphatischen Leukämie des Kindesalters ist die Gabe von anti-PD-1 bislang Einzelfällen vorbehalten [9].

Zusammenfassend ist die Immuntherapie der ALL ein beispielloser Erfolg der aktuellen Therapieentwicklung. Sowohl Antikörper als auch zelluläre Therapien wurden entwickelt. Gemeinsam ist beiden, dass die Wirkung auf der Funktion von T-Zellen aufbaut. Die pädiatrische AML wird erst noch präklinische Forschung zur erfolgreichen Nutzung dieses Effektes benötigen. Der klinische Stellenwert der Immuntherapie in der Behandlung kindlicher Leukämien wird sich in den nächsten Jahren in klinischen Studien beweisen müssen, um die exakte Indikation und Patientenpopulation zu kennen, welche optimal von diesen Therapien profitieren wird. 
Die Autoren geben an, dass kein Interessenkonflikt besteht.

\section{Autor}

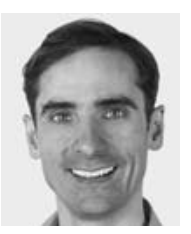

Tobias Feuchtinger

Dr. von Haunersches Kinder-

spital, Ludwig-Maximilians-

Universität, München

\section{Korrespondenzadresse}

Prof. Dr. med. Tobias Feuchtinger

Leiter der Abteilung für Pädiatrische Hämatologie, Onkologie, Hämostaseologie und Stammzelltransplantation

Dr. von Haunersches Kinderspital Ludwig-Maximilians-Universität Lindwurmstraße 4

80337 München

tobias.feuchtinger@med.uni-muenchen.de
[1] Hunger SP, Lu X, Devidas M et al. Improved survival for children and adolescents with acute lymphoblastic leukemia between 1990 and 2005: a report from the children's oncology group. J Clin Oncol 2012; 30: 1663-1669

[2] Chen L, Flies DB. Molecular mechanisms of T cell co-stimulation and co-inhibition. Nat Rev Immunol 2013; 13: 227-242

[3] Nirschl C], Drake CG. Molecular pathways: coexpression of immune checkpoint molecules: signaling pathways and implications for cancer immunotherapy. Clin Cancer Res 2013; 19: 4917-4924

[4] Hamid O, Robert C, Daud A et al. Safety and tumor responses with lambrolizumab (antiPD-1) in melanoma. N Engl J Med 2013; 369: 134-144

[5] Hodi FS, O'Day S], McDermott DF et al. Improved survival with ipilimumab in patients with metastatic melanoma. $\mathrm{N}$ Engl J Med 2010; 363: 711-723
[6] Topp MS, Gokbuget N, Stein AS et al. Safety and activity of blinatumomab for adult patients with relapsed or refractory B-precursor acute lymphoblastic leukaemia: a multicentre, single-arm, phase 2 study. Lancet Oncol. 2015; 16: 57-66

[7] Maude SL, Frey N, Shaw PA et al. Chimeric antigen receptor $\mathrm{T}$ cells for sustained remissions in leukemia. N Engl J Med 2014; 371: 1507-1517

[8] Lee DW, Kochenderfer JN, Stetler-Stevenson $\mathrm{M}$ et al. T cells expressing CD19 chimeric antigen receptors for acute lymphoblastic leukaemia in children and young adults: a phase 1 dose-escalation trial. Lancet 2015; 385: 517-528

[9] Boekstegers AM, Blaeschke F, Schmid I et al. MRD response in a refractory paediatric T-ALL patient through anti-programmed cell death 1 (PD-1) Ab treatment associated with induction of fatal GvHD. Bone Marrow Transplant 2017; 52: 1221-1224 